\section{Rheumatoide Arthritis: Knochenschwund unter Kortisontherapie ist vermeidbar}

Rheumapatienten zusätzlich zu Methotrexat ein Glukokortikoid zu verordnen, muss nicht zwangsläufig zulasten der Knochen gehen - vorausgesetzt, es wird eine umfassende Osteoporoseprophylaxe durchgeführt. Dies legen zumindest die Ergebnisse der CAMERA-II-Studie nah.

\footnotetext{
In der CAMERA-II-Studie wurden bei Patienten mit neu diagnostizierter rheumatoider Arthritis (RA) über zwei Jahre hinweg die Knochendichte gemessen. Alle 236 Patienten, über die Hälfte von ihnen Frauen, erhielten eine kontinuierlich an die Krankheitsaktivität angepasste Basistherapie mit Methotrexat (MTX), Vitamin D, Kalzium sowie ein Bisphosphonat zur Osteoporoseprophylaxe. Zusätzlich wurden die Studienteilnehmer randomisiert mit $10 \mathrm{mg} / \mathrm{d}$ Prednison oder Placebo behandelt.

Durch die zusätzliche Behandlung mit dem Glukokortikoid konnte die Kontrolle der Krankheitsaktivität verbessert und die Gelenkdestruktion aufgehalten wer-
}

dem. Dieser Behandlungserfolg ging nicht zulasten der Knochendichte, wie DXA-Messungen bei $61 \%$ der Patienten belegen: An der Lendenwirbeslsäule (LWS) zeigte sich sogar in beiden Behandlungsgruppen ein signifikanter Anstieg der Knochendichte gegenüber dem Ausgangszustand, und zwar um 2,7\% in der Prednisongruppe und um 2,4\% unter Placebo nach einem Jahr sowie um 2,4\% respektive $3,4 \%$ nach zwei Jahren. An der Hüfte fanden sich hingegen keine signifikanten Veränderungen der Knochendichte. Weder an der Hüfte noch an der LWS bestanden zu irgendeinem Zeitpunkt signifikante Unterschiede zwischen Prednison und Placebo.
Regressionsanalysen bestätigten, dass die Therapiestrategie - mit oder ohne Glukokortikoid - keinen Einfluss auf die Entwicklung der Knochendichte hatte. Dagegen waren höheres Alter und geringeres Gewicht sowie höhere Krankheitsaktivität mit einer niedrigeren Knochendichte assoziiert.

Fazit: „Auf die Gabe von 10 mg Prednison pro Tag in der Frühphase einer RA sollte man nicht aus Angst vor einer steroidinduzierten Osteoporose verzichten", lautet das Fazit der Studienautoren um M. C. van der Goes, Universität Utrecht. Erhalten die Patienten eine Osteoporoseprophylaxe inklusive eines Bisphosphonats, sei zumindest in den ersten zwei Jahren kein Verlust an Knochendichte zu erwarten.

Dr. Beate Schumacher

Van der Goes MC et al. Are changes in bone mineral density different between groups of early rheumatoid arthritis patients treated according to a tight control strategy with or without prednisone if osteoporosis prophylaxis is applied? Osteoporos Int 2012;

Doi: 10.1007/s00198-012-2073-z

\section{Chronische Schmerzen: Besorgte Lebensgefährten lassen die Opioiddosis steigen}

Sind die Lebensgefährten allzu besorgt um ihren von chronischen Schmerzen geplagten Partner, führt dies zu signifikant erhöhtem Opioidverbrauch. Das haben Schmerzmediziner herausgefunden und eine direkte Korrelation zwischen dem Ausmaß der Sorge und der Opioidmenge errechnet.

F ür ihre Studie haben die Forscher die Angaben von 466 konsekutiven Patienten retrospektiv analysiert, die zwischen September 2003 und Februar 2007 wegen chronischer, nicht durch Krebs verursachter Schmerzen ein Rehabilitationsprogramm durchlaufen hatten.

Die Teilnehmer hatten dabei auch das Multidimensional Pain Inventory beantwortet. Darin wird u.a. gefragt, wie besorgt der Partner auf die Schmerzen reagiert - etwa ob er den Schmerzen viel Beachtung schenkt, wie viel Unterstützung er gewährt und wie groß seine Sorgen sind. Dabei können null bis sechs Punkte vergeben werden; je höher die Punktzahl, desto größer die Sorge. Für die Studie wurde die auf den Partner be- zogene Subskala standardisiert, sodass ihr Bereich von 0 bis 100 reichte.

Im Mittel erreichte der Punktwert der Partnersorge 49,8. Die mittlere standardisierte Morphindosis lag bei $118 \mathrm{mg} / \mathrm{Tag}$. In der multivariaten Analyse - abgeglichen z.B. nach Alter, Geschlecht, Schmerzdauer und -intensität - stellte sich heraus, dass ein Anstieg um einen Punkt auf der Skala eine Erhöhung der täglichen Morphinäquivalenzdosis um 2,1 mg nach sich zog. Auch jüngeres Alter und männliches Geschlecht waren mit höherem Opioidverbrauch assoziiert.

Die Wissenschaftler fordern als Kon-

Patienten mit besorgten Angehörigen haben einen höheren Opioidverbrauch. sequenz aus ihren Ergebnissen, die Lebensgefährten von Schmerzpatienten in eine kognitive Verhaltenstherapie einzubeziehen. In diesen Therapien sollen die Patienten - und eben auch ihre Partner - lernen, wie chronische Schmerzen bewältigt werden können. So sollen die schädlichen Wirkungen gemildert werden, die von besorgten Reaktionen des Partners ausgehen.

Dr. Robert Bublak

Cunningham J et al. Associations Between Spousal or Significant Other Solicitous Responses and Opioid Dose in Patients with Chronic Pain. Pain Med. 2012;13(8):1034-9.

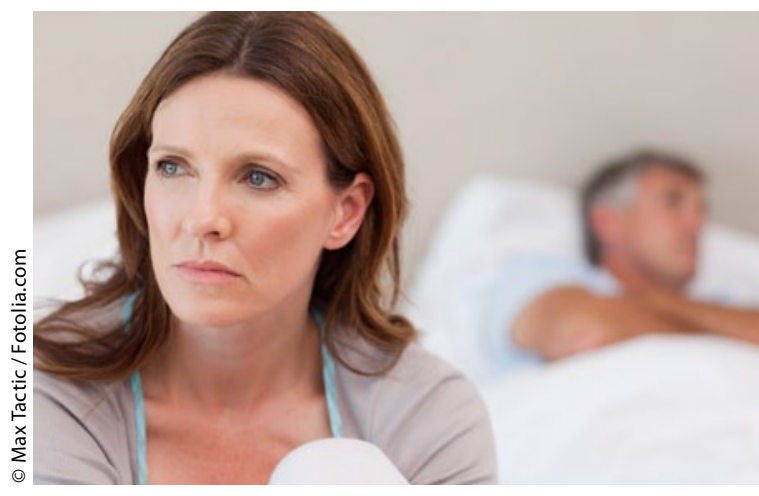

